International Journal of Pure and Applied Mathematics

Volume 90 No. 2 2014, 177-188

ISSN: 1311-8080 (printed version); ISSN: 1314-3395 (on-line version)

url: http://www.ijpam.eu

doi: http://dx.doi.org/10.12732/ijpam.v90i2.7

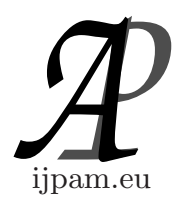

\title{
FINITE SERIES AND COMPLETE SOLUTION OF SECOND ORDER $\alpha$-DIFFERENCE EQUATION
}

\author{
G. Britto Antony Xavier ${ }^{1}$, P. Rajiniganth ${ }^{2}$, V. Chandrasekar ${ }^{3}$ \\ ${ }^{1}$ Department of Mathematics \\ Sacred Heart College \\ Tirupattur, 635601, Vellore District, Tamil Nadu, S. INDIA \\ 2 Department of Mathematics \\ SKP Institute of Technology \\ Tiruvannamalai, 606611, Tamil Nadu, S. INDIA \\ ${ }^{3}$ Department of Mathematics \\ SKP Engineering College \\ Tiruvannamalai, 606611, Tamil Nadu, S. INDIA
}

\begin{abstract}
We derive the discrete version of Leibnitz Theorem, Montmorte's Theorem with respect to generalized $\alpha$-difference equation. We also investigate the numerical and complete solutions of second order $\alpha$-difference equation for finding the values of various finite $\alpha$-series in the field of finite difference method.
\end{abstract}

AMS Subject Classification: 39A70, 47B39, 39A10

Key Words: generalized $\alpha$-difference equation, numerical solution, complete solution, generalized polynomial factorial

\section{Introduction}

In 1984, Jerzy Popenda[4] while discussing the behavior of solutions a particular type of difference equation defined $\Delta$ as $\Delta_{\alpha} u(k)=u(k+1)-\alpha u(k)$. In 1989

Received: September 18, 2013

(c) 2014 Academic Publications, Ltd. url: www.acadpubl.eu

$\S_{\text {Correspondence author }}$ 
Miller and Rose[14] introduced the discrete analogue of the Riemann-Liouville fractional derivative and proved some properties of the fractional difference operator. In the general fractional h-difference Riemann-Liouville operator mentioned in $[15,16]$, the presence of the $\mathrm{h}$ parameter is particularly interesting from the numerical point of view, because when $\mathrm{h}$ tends to zero the solutions of fractional difference equations can be seen as approximations to the solutions of corresponding Riemann-Liouville fractional differential equation $[15,17]$. On the otherhand, fractional h sum of order $m \geq 1\left(\left(\Delta_{h}^{-m} f\right)(t)\right.$ definition 2.8 of $[15])$ is very useful to derive many interesting results in a different way in number theory such as the sum of the $m^{\text {th }}$ partial sums on $n^{\text {th }}$ powers of arithmetic, arithmetic-geometric progressions, and products of $\mathrm{n}$ consecutive terms of arithmetic progression using $\Delta_{\ell}^{-m} u(k)$ [10]. This definition of $\Delta$ is being ignored for a long time. In 2011, M.Maria Susai Manuel, et.al, [11] have extended the definition of $\Delta_{\alpha}$ to $\Delta_{\alpha(\ell)}$ defined as $\Delta_{\alpha(\ell)} u(k)=u(k+\ell)-\alpha u(k)$ for the real valued function $u(k)$ and $\ell \in(0, \infty)$. In [12], the authors have used the generalized $\alpha$-difference equation;

$$
v(k+\ell)-\alpha v(k)=u(k), k \in[0, \infty), \ell \in(0, \infty)
$$

and obtained a numerical solution of the above equation in the form

$$
v(k)=\left.\Delta_{\alpha(\ell)}^{-1} u(k)\right|_{j} ^{k}=\sum_{r=1}^{\left[\frac{k}{\ell}\right]} \alpha^{r-1} u(k-r \ell), \quad j=k-\left[\frac{k}{\ell}\right] \ell .
$$

There are two type of solutions for the equation (3); one is numerical another one is closed form solution. If we are able to find a closed form solution of equation (3), which coinciding with the numerical solution of that equation, then we can obtain formula of values of several finite series. In this paper, we extend the theory and applications of $\Delta_{\alpha(\ell)}$ developed in [12] to generalized second kind $\alpha$-difference equation

$$
\Delta_{\alpha(\ell, m)} v(k) \equiv v(k+\ell+m)-\alpha[v(k+\ell)+v(k+m)]+\alpha^{2} v(k)=u(k),
$$

where $\alpha, \ell, m \in(0, \infty)$ and $k \geq 0$ is variable, and investigate numerical - complete solutions of eqution of (3) to obtain the values of several finite series. When $\alpha=1$, the results of this paper coincides with [9]. 


\section{Preliminaries}

Before stating and proving our results, we present some notations, basic definitions and preliminary results which will be useful for further subsequent discussions. Let $\ell>0, k \in[0, \infty), j=k-\left[\frac{k}{\ell}\right] \ell$ where $\left[\frac{k}{\ell}\right]$ denotes the integer part of $\frac{k}{\ell}, E^{\ell} v(k)=v(k+\ell), \Delta_{\alpha(\ell)} v(k)=v(k+\ell)-\alpha v(k), \mathbb{N}_{\ell}(j)=\{j, \ell+j, 2 \ell+j, \cdots\}$ and $\mathbb{N}_{1}(j)=\mathbb{N}(j)$. Throughout this paper, $c_{0 j}, c_{1 j}$ are constants for all $k \in \mathbb{N}_{\ell}(j)$ and $\alpha \neq 0$ and 1 .

The following is the product and quotient rule of $\Delta_{\alpha(\ell, m)}$.

Lemma 2.1. Let $u(k)$ and $v(k) \neq 0$ be two real valued functions. Then, (i) $\Delta_{\alpha(\ell, m)}[u(k) v(k)]=u(k) \Delta_{\alpha(\ell, m)} v(k)+\left[v(k+\ell+m) \Delta_{\ell+m}-\right.$ $\left.\alpha\left(v(k+\ell) \Delta_{\ell}+v(k+m) \Delta_{\alpha(m)}\right)\right] u(k)$.

$$
\begin{gathered}
(i i) \quad \Delta_{\alpha(\ell, m)} \frac{u(k)}{v(k)}=\frac{1}{v(k) v(k+\ell) v(k+m) v(k+\ell+m)}\{v(k+\ell) \\
v(k+m)\left[v(k) \Delta_{\alpha(\ell+m)} u(k)-u(k) \Delta_{\alpha(\ell+m)} v(k)\right]-v(k+\ell+m) \\
\left\{\alpha v(k+m)\left[v(k) \Delta_{\alpha(\ell)} u(k)-\alpha u(k) \Delta_{\alpha(\ell)} v(k)\right]+v(k+\ell)[v(k)\right. \\
\left.\left.\left.\alpha \Delta_{\alpha(m)} u(k)-u(k) \Delta_{\alpha(m)} v(k)\right]\right\}\right\} .
\end{gathered}
$$

Proof. The proof follows from $\Delta_{\alpha(\ell, m)}=\Delta_{\alpha(\ell)}\left(\Delta_{\alpha(m)}\right)$ and the expression of $\Delta_{\alpha(\ell)}$ and $\Delta_{\alpha(m)}$.

The following can be obtained by simple algebraic derivations.

Lemma 2.2. The relations among $E^{\ell}$ and the difference operators $\Delta$, $\Delta_{\alpha(\ell)}, \Delta_{\alpha(m)}$ and $\Delta_{\alpha(\ell, m)}$ are given as:

$$
\begin{gathered}
\text { (i) } \Delta_{\alpha(\ell, m)}=E^{\ell+m}-\alpha\left[E^{\ell}+E^{m}\right]+\alpha^{2}, \\
\text { (ii) } \Delta_{\alpha(\ell, m)}=\Delta_{\alpha(\ell+m)}-\alpha\left[\Delta_{\alpha(\ell)}+\Delta_{\alpha(m)}\right]+\left(\alpha-\alpha^{2}\right), \\
\text { (iii) } \Delta_{\alpha(\ell, m)}=\left(\sum_{i=1}^{\ell} \ell C_{i} \Delta^{i}-\alpha\right)\left(\sum_{j=1}^{m} m C_{j} \Delta^{j}-\alpha\right), \ell, m \in \mathbb{N}(1) .
\end{gathered}
$$

(iv) If $\ell_{i}$ and $m_{i}$ are positive integers with $\ell=\sum_{i=1}^{n} \ell_{i}$ and $m=\sum_{i=1}^{n} m_{i}$, then $\Delta_{\alpha(\ell, m)}=\left[\prod_{i=1}^{n}\left(\Delta_{\alpha\left(\ell_{i}\right)}+\alpha\right)-\alpha\right]\left[\prod_{i=1}^{n}\left(\Delta_{\alpha}\left(m_{i}\right)+\alpha\right)-\alpha\right]$. 
(v) $\Delta_{\alpha(n \ell, n m)}=E^{n(\ell+m)}-\alpha\left[E^{n \ell}+E^{n m}\right]+\alpha^{2}$.

(vi) $E^{n(\ell+m)}-\alpha\left[E^{n \ell}+E^{n m}\right]=\sum_{r=0}^{n} \alpha^{r} n C_{r}$

$$
\left[\sum_{i=0}^{n-1}(-\alpha)^{i} n C_{i}\left(\Delta_{\alpha(n-i)(\ell+m)}\right)-\alpha\left(\Delta_{\alpha(n-i) \ell}+\Delta_{\alpha(n-i) m}\right)\right] .
$$

The following are easy deductions.

Lemma 2.3. For $n \in \mathbb{N}(2)$, let $\Delta_{\alpha(\ell, m)}^{n}=\Delta_{\alpha(\ell, m)}\left(\Delta_{\alpha(\ell, m)}^{n-1}\right)$. Then,

$$
\text { (i) } \Delta_{\alpha(\ell, m)}^{r}=\left(\sum_{i=0}^{r}(-\alpha)^{i} r C_{i} E^{\ell(r-i)}\right)\left(\sum_{j=0}^{r}(-\alpha)^{j} r C_{j} E^{m(r-j)}\right)
$$

and hence for the function $u(k)$,

$$
\begin{aligned}
& \Delta_{\alpha(\ell, m)}^{r} u(k)=\sum_{i=0}^{r}(-\alpha)^{i} r C_{i} u(k+\ell(r-i)) \sum_{j=0}^{r}(-\alpha)^{j} r C_{j} u(k+m(r-j)) \\
& \text { (ii) } \Delta_{\alpha(n \ell, n m)}=\left(\alpha+\Delta_{\alpha(\ell+m)}\right)^{n}-\alpha\left[\left(\alpha+\Delta_{\alpha(\ell)}\right)^{n}+\left(\alpha+\Delta_{\alpha(m)}\right)^{n}\right]+\alpha^{2} . \\
& \text { (iii) } \Delta_{\alpha(n \ell, n m)}=\sum_{r=0}^{n} n C_{r} \alpha^{n-r}\left[\Delta_{\alpha(\ell+m)}^{r}-\alpha\left(\Delta_{\alpha(\ell)}^{r}+\Delta_{\alpha(m)}^{r}\right)\right]+\alpha^{2} \\
& \text { (iv) } \Delta_{\alpha(\ell, m)}^{n}=\sum_{r=0}^{n} \sum_{i=0}^{n} \sum_{j=0}^{r} n C_{r}\left(\alpha-\alpha^{2}\right)^{n-r} n C_{i}(-\alpha)^{i} \Delta_{\alpha(\ell+m)}^{n-i} n C_{j} \Delta_{\alpha(\ell)}^{r-j} \Delta_{\alpha(m)}^{j} \\
& \text { (v) } \Delta_{\alpha(\ell, m)}^{n}=\left(\sum_{i=0}^{n-1}(-\alpha)^{i} n C_{i} \Delta_{\alpha(n-i) \ell}\right)\left(\sum_{j=0}^{n-1}(-\alpha)^{j} n C_{j} \Delta_{\alpha(n-j) m}\right) .
\end{aligned}
$$

Proof. The proof follows from the expressions of corresponding notations and simple arithmetic derivations.

Following is the discrete version of Leibnitz theorem on $\Delta_{\alpha(\ell, m)}$.

Theorem 2.4. Let $u(k)$ and $v(k) \neq 0$ be two real valued functions. Then, $\Delta_{\alpha(\ell, m)}^{n}[u(k) v(k)]=\Delta_{\alpha(m)}^{n}\left[u(k) \alpha^{n} \Delta_{\alpha(\ell)}^{n} v(k)\right]+n C_{1} \Delta_{\alpha(m)}^{n}$

$$
\begin{gathered}
{\left[\Delta_{\alpha(\ell)} u(k) \alpha^{n-1} \Delta_{\alpha(\ell)}^{n-1} v(k+\ell)\right]+n C_{2} \Delta_{\alpha(m)}^{n}\left[\Delta_{\alpha(\ell)}^{2} u(k) \alpha^{n-2}\right.} \\
\left.\Delta_{\alpha(\ell)}^{n-2} v(k+2 \ell)\right]+\cdots+n C_{n} \Delta_{\alpha(m)}^{n}\left[\Delta_{\alpha(\ell)}^{n} u(k) v(k+n \ell)\right] .
\end{gathered}
$$

Proof. The proof follows by the generalized Leibnitz Theorem (Theorem $2.5,[8])$ and the relation $\Delta_{\alpha(\ell, m)}=\Delta_{\alpha(\ell)} \Delta_{\alpha(m)}$. 
Theorem 2.5. Let $n, p \in \mathbb{N}(1)$. Then,

$$
\begin{aligned}
& (k+n \ell+n m)^{p}-\alpha\left[(k+n \ell)^{p}+(k+n m)^{p}\right]=\sum_{r=0}^{n} \sum_{s=0}^{r-1} \alpha^{r} n C_{r}(-1)^{s} r C_{s} \\
& \left\{[k+(r-s)(\ell+m)]^{p}-\alpha\left[(k+(r-s) \ell)^{p}+(k+(r-s) m)\right]^{p}-(2 \alpha-1) k^{p}\right\} .
\end{aligned}
$$

Proof. The proof is achieved by operating $u(k)=k^{p}$ on (7) and using the relation $\Delta_{\alpha(\ell)}^{r} k^{p}=\sum_{i=0}^{r}(-\alpha)^{i} r C_{i}(k+\ell(r-i))^{p}$.

Lemma 2.6. Let $u(k)$ be real valued function on $[0, \infty)$ and $x \geq 0$. Then

$$
\begin{aligned}
& \sum_{j=0}^{\infty}\left[\frac{x^{j(\ell+m)} u(j(\ell+m))}{j !(\ell+m)^{j}}-\alpha\left(\frac{x^{j \ell} u(j \ell)}{j ! \ell^{j}}+\frac{x^{j m} u(j m)}{j ! m^{j}}\right)\right] \\
= & {\left[e^{\frac{x^{\ell+m} \alpha}{\ell+m}} e^{\frac{x^{\ell+m} \Delta_{\alpha(\ell+m)}}{\ell+m}}-\alpha\left(e^{\frac{\alpha x^{\ell}}{\ell}} e^{\frac{x^{\ell} \Delta_{\alpha(\ell)}}{\ell}}+e^{\frac{\alpha x^{m}}{m}} e^{\frac{x^{m} \Delta_{\alpha(m)}}{m}}\right)\right] u(0) . }
\end{aligned}
$$

Proof. Proof follows by (4), $u(k)=E^{k} u(0) \& \alpha+\Delta_{\alpha(\ell)}=E^{\ell}$.

Lemma 2.7. [8] If $s_{r}^{n}$ and $S_{r}^{n}$ are the Stirling numbers of the first and second kinds and $k_{\ell}^{(n)}=k(k-\ell)(k-2 \ell) \ldots(k-(r-1) \ell)$, then

$$
k_{\ell}^{(n)}=\sum_{r=1}^{n} s_{r}^{n} \ell^{n-r} k^{r} \quad \text { and } \quad k^{n}=\sum_{r=1}^{n} S_{r}^{n} \ell^{n-r} k_{\ell}^{(r)} .
$$

\section{Numerical - Complete Solutions of (3)}

In this section, a numerical solution and a complete solution of the equation (3) have been derived, to obtain the value of several finite series in the field of finite difference method.

Definition 3.1. A function $v(k)$ satisfying $\alpha$-difference equation $v(k+$ $\ell)-\alpha v(k)=u(k)$ is called a solution of the equation and it can be denoted as $v(k)=\Delta_{\alpha(\ell)}^{-1} u(k)$. Similarly, $v(k)$ satisfying equation (3) is called solution of that equation and is denoted as $v(k)=\Delta_{\alpha(\ell, m)}^{-1} u(k)$. 
Lemma 3.2. If $v(k)$ is any closed form solution of the equation (3) then

$$
w_{1}(k)=\left\{\begin{array}{ccc}
v(k)-\frac{\alpha^{\left[\frac{k}{\ell}\right]}}{\alpha^{\left[\frac{m}{\ell}\right]}-\alpha} c_{0 j}-\frac{\alpha^{\left[\frac{k}{m}\right]}}{\alpha^{\left[\frac{\ell}{m}\right]}-\alpha} c_{1 j} & \text { for } & \ell \neq m \\
v(k)-\alpha^{\left[\frac{k}{\ell}\right]-1} v(j) & \text { for } & \ell=m .
\end{array}\right.
$$

is also a solution of the equation (3). Now $w_{1}(k)$ is called as complete solution of the equation (3).

Lemma 3.3. Let $0<\ell<k$ and $\left[\frac{k}{\ell}\right]=$ integer part of $\frac{k}{\ell}$. Then, when $\ell=m$ in $(3)$

$$
v(k)=\sum_{r=2}^{\left[\frac{k}{\ell}\right]}(r-1) \alpha^{r-2} u(k-r \ell)
$$

satisfies the generalized second order $\alpha$-difference equation

$$
v(k+2 \ell)-2 \alpha v(k+\ell)+\alpha^{2} v(k)=u(k) .
$$

Hence (13)is called as a numerical solution of the equation (14).

Lemma 3.4. Let $P_{k}$ be any function of $k$ on $[0, \infty)$, then

$$
\begin{aligned}
v(k)=\frac{\lambda^{k}}{\left(\lambda^{\ell}-\alpha\right)^{2}} & \sum_{t=0}^{\infty}(-1)^{t}(t+1)\left[\frac{\lambda^{\ell} \Delta(\ell)}{\left(\lambda^{\ell}-\alpha\right)}\right]^{t} P_{k} \\
& -\left.\left.\left\{\left[\frac{k}{\ell}\right] \alpha\left[\frac{k}{\ell}\right]-1 \frac{\lambda^{j}}{\left(\lambda^{\ell}-\alpha\right)} \sum_{t=0}^{\infty}(-1)^{t}\left[\frac{\lambda^{\ell} \Delta(\ell)}{\left(\lambda^{\ell}-\alpha\right)}\right]^{t} P_{k}\right\}\right|_{j}\right|_{\ell+j} ^{k}
\end{aligned}
$$

is a complete solution of the equation (14), for $u(k)=\lambda^{k} P_{k}$.

Proof. From the generalized $\alpha$-difference operator, we find that

$$
\begin{array}{r}
\Delta_{\alpha(\ell)} \lambda^{k} F_{k}=\lambda^{k+\ell} F_{(k+\ell)}-\alpha \lambda^{k} F_{k} \\
=\lambda^{k}\left(\lambda^{\ell} E^{\ell}-\alpha\right) F_{k}=\lambda^{k} P_{k},
\end{array}
$$

where $P_{k}=\left(\lambda^{\ell} E^{\ell}-\alpha\right) F_{k}$ (or) $F_{k}=\left(\lambda^{\ell} E^{\ell}-\alpha\right)^{-1} P_{k}$, yields

$$
\begin{aligned}
\Delta_{\alpha(\ell)}^{-1} \lambda^{k} P_{k} & =\lambda^{k} F_{k}=\lambda^{k}\left(\lambda^{\ell} E^{\ell}-\alpha\right)^{-1} P_{k} \\
& =\frac{\lambda^{k}}{\left(\lambda^{\ell}-\alpha\right)}\left[1-\frac{\lambda^{\ell}}{\left(\lambda^{\ell}-\alpha\right)} \Delta_{\ell}+\frac{\lambda^{2 \ell}}{\left(\lambda^{\ell}-1\right)^{2}} \Delta_{\ell}^{2}+\ldots\right] P_{k}
\end{aligned}
$$

and applying the limit from $j$ to $k$ we obtain

$$
\left.\Delta_{\alpha(\ell)}^{-1} \lambda^{k} P_{k}\right|_{j} ^{k}=\left.\frac{\lambda^{k}}{\left(\lambda^{\ell}-\alpha\right)} \sum_{t=0}^{\infty}(-1)^{t}\left[\frac{\lambda^{\ell} \Delta_{\ell}}{\left(\lambda^{\ell}-\alpha\right)}\right]^{t} P_{k}\right|_{j} ^{k},
$$


which is a complete solution of the generalized first order $\alpha$ - difference equation $v(k+\ell)-\alpha v(k)=\lambda^{k} P_{k}$. Again taking $\Delta_{\alpha(\ell)}^{-1}$ in the above expression and taking limit from $\ell+j$ to $k$, we get (15).

Lemma 3.5. If $k \geq 2 \ell$, then $v(k)=\sum_{r=2}^{\left[\frac{k}{\ell}\right]}(r-1) \alpha^{r-2} \lambda^{k-r \ell} P_{k-r \ell}$ is a numerical solution of (14) for the function $u(k)=\lambda^{k} P_{k}$.

Proof. From the inverse of generalized $\alpha$ - difference operator and taking the limit from $j$ to $k$, we find $\Delta_{\alpha(\ell)}^{-1} \lambda^{k} P_{k}=\sum_{r=1}^{\left[\frac{k}{\ell}\right]} \alpha^{r-1} \lambda^{k-r \ell} P_{k-r \ell}$ which is a numerical solution of the generalized first order $\alpha$ - difference equation $v(k+\ell)-\alpha v(k)=$ $\lambda^{k} P_{k}$. Again taking $\Delta_{\alpha(\ell)}^{-1}$ in the above expression, applying the limit from $\ell+j$ to $k$ and using definition (3.3), we get the proof.

Theorem 3.6. Let $P_{k}$ be any function of $k$ on $[0, \infty)$. Then,

$$
\begin{array}{r}
\sum_{r=2}^{\left[\frac{k}{\ell}\right]}(r-1) \alpha^{r-2} \lambda^{k-r \ell} P_{k-r \ell}=\left.\frac{\lambda^{k}}{\left(\lambda^{\ell}-\alpha\right)^{2}} \sum_{t=0}^{\infty}(-1)^{t}(t+1)\left[\frac{\lambda^{\ell} \Delta_{(\ell)}}{\left(\lambda^{\ell}-\alpha\right)}\right]^{t} P_{k}\right|_{\ell+j} ^{k} \\
-\left[\frac{k}{\ell}\right] \alpha\left[\frac{k}{\ell}\right]-\left.\left.1\left\{\frac{\lambda^{j}}{\left(\lambda^{\ell}-\alpha\right)} \sum_{t=0}^{\infty}(-1)^{t}\left[\frac{\lambda^{\ell} \Delta_{(\ell)}}{\left(\lambda^{\ell}-\alpha\right)}\right]^{t} P_{k}\right\}\right|_{j}\right|_{\ell+j} ^{k} .
\end{array}
$$

Proof. The proof follows by equating the lemma (3.4) and (3.5)

In the following theorem the formula for sum of all partial sums of a finite arithmetic factorial-geometric series is obtained by equating Numerical and Complete solution of equation 14

Theorem 3.7. Consider all the terms in Theorem 3.6. Then,

$$
\begin{gathered}
\sum_{r=2}^{\left[\frac{k}{\ell}\right]}(r-1) \alpha^{r-2} \lambda^{k-r \ell}(k-r \ell)_{\ell}^{(n)}=\left.\sum_{t=0}^{n}(-1)^{t}(t+1) n^{(t)} \frac{\ell^{t} \lambda^{k+t \ell} k_{\ell}^{(n-t)}}{\left(\lambda^{\ell}-\alpha\right)^{t+2}}\right|_{\ell+j} ^{k} \\
-\left[\frac{k}{\ell}\right] \alpha\left[\frac{k}{\ell}\right]-\left.\left.1\left\{\sum_{t=0}^{n}(-1)^{t} n^{(t)} \ell^{t} \frac{\lambda^{j+t \ell}}{\left(\lambda^{\ell}-\alpha\right)^{t+1}} k_{\ell}^{(n-t)}\right\}\right|_{j} ^{k}\right|_{\ell+j} ^{k}
\end{gathered}
$$

Proof. The proof follows by taking $P_{k}=k_{\ell}^{(n)}$ in Theorem (3.6).

The following example is illustration of Theorem 3.7, when $n=3$. 
Example 3.8. In (17), by taking $n=3$, we obtain

$$
\begin{gathered}
\sum_{r=2}^{\left[\frac{k}{\ell}\right]}(r-1) \alpha^{r-2} \lambda^{k-r \ell}(k-r \ell)_{\ell}^{(3)}=\left.\sum_{t=0}^{3}(-1)^{t}(t+1) 3^{(t)} \frac{\ell^{t} \lambda^{k+t \ell} k_{\ell}^{(3-t)}}{\left(\lambda^{\ell}-\alpha\right)^{t+2}}\right|_{\ell+j} ^{k} \\
-\left[\frac{k}{\ell}\right] \alpha\left[\frac{k}{\ell}\right]-\left.\left.1\left\{\sum_{t=0}^{3}(-1)^{t} 3^{(t)} \ell^{t} \frac{\lambda^{j+t \ell}}{\left(\lambda^{\ell}-\alpha\right)^{t+1}} k_{\ell}^{(3-t)}\right\}\right|_{j} ^{k}\right|_{\ell+j} ^{k}
\end{gathered}
$$

In particular $k=61, \ell=3, \alpha=2$ and $\lambda=2$ we get,

$$
\begin{aligned}
& \sum_{r=2}^{20}(r-1) 2^{r-2} 2^{61-3 r}(61-3 r)_{3}^{(3)}=\left.\sum_{t=0}^{3}(-1)^{t}(t+1) 3^{(t)} \ell^{t} \frac{2^{k+3 t}}{6^{t+2}} k_{3}^{(3-t)}\right|_{4} ^{61} \\
& -\left[\frac{k}{\ell}\right] \alpha\left[\frac{k}{\ell}\right]-\left.1\left\{\sum_{t=0}^{3}(-1)^{t} 3^{(t)} 3^{t} \frac{2^{1+2 t}}{6^{t+1}}(1)_{3}^{(3-t)}\right\}\right|_{4} ^{61} \\
& =8051875685785775636480 \text {. }
\end{aligned}
$$

Following is a formula for sum all partial sums of arithmetic power-geometric series.

Theorem 3.9. Consider all the terms in Theorem 3.6. Then,

$$
\begin{gathered}
\sum_{r=2}^{\left[\frac{k}{\ell}\right]}(r-1) \alpha^{r-2} \lambda^{k-r \ell}(k-r \ell)^{n}=\sum_{t=0}^{n}(-1)^{t}(t+1) \\
\left.\frac{n^{(t)} \ell^{t} \lambda^{k+t \ell}}{\left(\lambda^{\ell}-\alpha\right)^{t+2}} \sum_{i=1}^{n-t} S_{i}^{n-t} \ell^{n-t-i} k_{\ell}^{(i)}\right|_{\ell+j} ^{k}-\left[\frac{k}{\ell}\right] \alpha\left[\frac{k}{\ell}\right]-1 \\
\left.\left.\left\{\sum_{t=0}^{n}(-1)^{t} n^{(t)} \ell^{t} \frac{\lambda^{j+t \ell}}{\left(\lambda^{\ell}-\alpha\right)^{t+1}} \sum_{i=1}^{n-t} S_{i}^{n-t} \ell^{n-t-i} k_{\ell}^{(i)}\right\}\right|_{j}\right|_{\ell+j} ^{k}
\end{gathered}
$$

Proof. Taking $P_{k}=k^{n}$ in (16) and using (11), we get proof.

The following example illustrates Theorem 3.9.

Example 3.10. If $\alpha \neq a^{\ell}, k \in[\ell, \infty)$ and $j=k-\left[\frac{k}{\ell}\right] \ell$, then

$$
\begin{aligned}
& \sum_{r=1}^{\left[\frac{k}{\ell}\right]}(r-1) \alpha^{r-2}(k-r \ell) a^{k-r \ell}=\frac{k a^{k}}{\left(a^{\ell}-\alpha\right)^{2}}-\frac{2 \ell a^{k+\ell}}{\left(a^{\ell}-\alpha\right)^{3}} \\
& -\left[\frac{k}{\ell}\right] \alpha\left[\frac{k}{\ell}\right]-\left.\left.1\left\{\frac{j a^{j}}{\left(a^{\ell}-\alpha\right)}-\frac{\ell a^{j+\ell}}{\left(a^{\ell}-\alpha\right)^{2}}\right\}\right|_{j}\right|_{\ell+j} ^{k} .
\end{aligned}
$$


Proof. The proof follows by taking $n=1$ and replacing $\lambda$ by $a$ in (18).

Example 3.11. Taking $k=27, \ell=2, a=5$ and $\alpha=7$ in (19), we obtain $\begin{aligned} & \sum_{r=1}^{\left[\frac{k}{2}\right]}(r-1) 7^{r-2}(k-r \ell) 5^{k-r \ell}=\frac{k 5^{k}}{(18)^{2}}-\frac{2.2 .5^{k+2}}{(18)^{3}}-\left[\frac{k}{2}\right] 7^{\left[\frac{k}{2}\right]-1} \\ &\left.\left\{\frac{5^{1}}{(18)}-\frac{2.5^{3}}{18^{2}}\right\}\right|_{3} ^{27}=493128372488834830 .\end{aligned}$

The following theorem is illustrates theorem 3.7, for $\lambda=1$.

Theorem 3.12. Let $k \in[\ell, \infty), j=k-\left[\frac{k}{\ell}\right] \ell$ and $n$ is a positive integer and $\alpha \neq 1$. Then,

$$
\begin{gathered}
\sum_{r=2}^{\left[\frac{k}{\ell}\right]}(r-1) \alpha^{r-2}( \\
(k-r \ell)_{\ell}^{(m)}=\left.\sum_{i=0}^{m} \frac{(-1)^{i}(i+1) m^{(i)} \ell^{i} k_{\ell}^{(m-i)}}{(1-\alpha)^{i+2}}\right|_{\ell+j} ^{k} \\
-\left.\left.\left[\frac{k}{\ell}\right] \alpha{ }^{\left[\frac{k}{\ell}\right]-1}\left\{\sum_{i=0}^{m} \frac{(-1)^{i} m^{(i)} \ell^{i} k_{\ell}^{(m-i)}}{(1-\alpha)^{i+1}}\right\}\right|_{j}\right|_{\ell+j} ^{k}
\end{gathered}
$$

Proof. The proof follows by taking $\lambda=1$ and $n=3$ in Theorem 3.7.

Corollary 3.13. Let $k \in[\ell, \infty), j=k-\left[\frac{k}{\ell}\right] \ell$ and $m=3$. Then

$$
\begin{gathered}
\sum_{r=2}^{\left[\frac{k}{\ell}\right]}(r-1) \alpha^{r-2}(k-r \ell)_{\ell}^{(3)}=\left.\sum_{i=0}^{3} \frac{(-1)^{i}(i+1) 3^{(i)} \ell^{i} k_{\ell}^{(3-i)}}{(1-\alpha)^{i+2}}\right|_{\ell+j} ^{k} \\
-\left[\frac{k}{\ell}\right] \alpha\left[\frac{k}{\ell}\right]-\left.\left.1\left\{\sum_{i=0}^{3} \frac{(-1)^{i} 3^{(i)} \ell^{i} k_{\ell}^{(3-i)}}{(1-\alpha)^{i+1}}\right\}\right|_{j} ^{k}\right|_{\ell+j} ^{k}
\end{gathered}
$$

The following example is an illustration of Corollary 3.13.

Example 3.14. By taking $\ell=3, \alpha=2$ and $k=211$ in (21), we obtain

$$
\begin{gathered}
\sum_{r=1}^{70}(r-1) 2^{r-2}(211-2 r)_{2}^{(3)}=\left.\sum_{i=0}^{3} \frac{(-1)^{i}(i+1) 3^{(i)} 3^{i} k_{3}^{(3-i)}}{(-1)^{i+2}}\right|_{3} ^{211} \\
-\left.\left.\left[\frac{k}{\ell}\right] \alpha^{\left[\frac{k}{\ell}\right]-1}\left\{\sum_{i=0}^{3} \frac{(-1)^{i} 3^{(i)} 2^{i} k_{3}^{(3-i)}}{(-1)^{i+1}}\right\}\right|_{1}\right|_{3} ^{211} \\
=7669123168180303836864158
\end{gathered}
$$


Theorem 3.15. Consider all the terms in Theorem 3.6. Then

$$
\begin{gathered}
\sum_{r=2}^{\left[\frac{k}{\ell}\right]}(r-1) \alpha^{r-2}(k-r \ell)^{n}=\left.\sum_{t=0}^{n} \frac{(-1)^{t}(t+1) n^{(t)}}{(1-\alpha)^{t+2}} \sum_{i=1}^{n-t} S_{i}^{n-t} \ell^{n-i} k_{\ell}^{(i)}\right|_{\ell+j} ^{k} \\
\quad-\left.\left[\frac{k}{\ell}\right] \alpha \alpha^{\left[\frac{k}{\ell}\right]-1}\left\{\left.\sum_{t=0}^{n} \frac{(-1)^{t} n^{(t)}}{(1-\alpha)^{t+1}} \sum_{i=1}^{n-t} S_{i}^{n-t} \ell^{n-i} k_{\ell}^{(i)}\right|_{j}\right\}\right|_{\ell+j} ^{k}
\end{gathered}
$$

Proof. Taking $P_{k}=k^{n}$ and $\lambda=1$ in (18) and using (11), we get proof.

The following corollary illustrates Theorem 3.15.

Corollary 3.16. Let $k \in[\ell, \infty), \alpha \neq 1, n=3$ and $j=k-\left[\frac{k}{\ell}\right] \ell$. Then

$$
\begin{aligned}
& \sum_{r=2}^{\left[\frac{k}{\ell}\right]}(r-1) \alpha^{r-2}(k-r \ell)^{3}=\left.\sum_{t=0}^{3} \frac{(-1)^{t}(t+1) 3^{(t)}}{(1-\alpha)^{t+2}} \sum_{i=1}^{3-t} S_{i}^{3-t} \ell^{3-i} k_{\ell}^{(i)}\right|_{\ell+j} ^{k} \\
& -\left[\frac{k}{\ell}\right] \alpha\left[\frac{k}{\ell}\right]-\left.\left.1\left\{\sum_{t=0}^{3} \frac{(-1)^{t} 3^{(t)}}{(1-\alpha)^{t+1}} \sum_{i=1}^{3-t} S_{i}^{3-t} \ell^{3-i} k_{\ell}^{(i)}\right\}\right|_{j}\right|_{\ell+j} ^{k}
\end{aligned}
$$

Proof. The proof follows by taking $n=3$ in (22)

The following example is an illustration of Corollary 3.16.

Example 3.17. Taking $k=70, \ell=3$ and $\alpha=7$ in (23), we obtain

$$
\begin{aligned}
\sum_{r=1}^{\left[\frac{k}{3}\right]}(r-1) 7^{r-2}(k-3 r)^{3} & =\left.\sum_{t=0}^{3} \frac{(-1)^{t}(t+1) 3^{(t)}}{(-6)^{t+2}} \sum_{i=1}^{3-t} S_{i}^{3-t} 3^{3-i} k_{3}^{(i)}\right|_{4} ^{70} \\
-\left[\frac{k}{\ell}\right] \alpha^{\left[\frac{k}{\ell}\right]-1} & \left.\left.\left\{\sum_{t=0}^{3} \frac{(-1)^{t} 3^{(t)}}{(-6)^{t+1}} \sum_{i=1}^{3-t} S_{i}^{3-t} 3^{3-i} k_{3}^{(i)}\right\}\right|_{1}\right|_{4} ^{70} \\
= & 1769085418343559978481
\end{aligned}
$$

\section{Acknowledgments}

Research Supported by University Grants Commission, SERO, Hyderabad, India. 


\section{References}

[1] R.P Agarwal, Difference Equations and Inequalities, Marcel Dekker, New York, 2000.

[2] R.P. Agarwal and P.J.Y. Wong, Advanced Topics in Difference Equations, Kluwer, Dordrecht, 1997.

[3] R.Almeida, D.F.M.Torres, Necessary and sufficient conditions for the fractional calculus of variations with Caputo derivatives, Commun. Non linear Sci. Numer. Simul. 16 (2011), no. 3,1490-1500.

[4] Jerzy Popenda and Blazej Szmanda, On the Oscillation of Solutions of Certain Difference Equations, Demonstratio Mathematica, XVII(1), (1984), $153-164$.

[5] Jerzy Popenda, Oscillation and Nonoscillation Theorems for Second-Order Difference Equations, Journal of Mathematical Analysis and Applications, 123(1), (1987), $34-38$.

[6] R.L.Magin, Fractional calculus in bioengineering, Begell House, 2006.

[7] A.B.Malinowska, D.F.M.Torres, Generalized natural boundary conditions for fractional variational problems in terms of the Caputo derivative, Comput. Math. Appl. 59 (9) (2010), 3110-3116.

[8] M.Maria Susai Manuel, G.Britto Antony Xavier and E.Thandapani, Theory of Generalized Difference Operator and Its Applications, Far East Journal of Mathematical Sciences, 20(2) (2006), 163 - 171.

[9] M.Maria Susai Manuel, G.Britto Antony Xavier and V.Chandrasekar, Theory of Generalized Difference Operator of the Second Kind and Its Applications in Number Theory, Far East Journal of Mathematical Sciences, 20(2) (2009), $163-171$.

[10] M. M. Susai Manuel, G.B. A. Xavier, V. Chandrasekar and R. Pugalarasu, Theory and application of the Generalized Difference Operator of the $n^{\text {th }}$ kind(Part I), Demonstratio Mathematica, 45 (2012),no.1, 95-106.

[11] M.Maria Susai Manuel, V.Chandrasekar and G.Britto Antony Xavier, Solutions and Applications of Certain Class of $\alpha$-Difference Equations, International Journal of Applied Mathematics, 24(6) (2011), 943-954. 
[12] M.Maria Susai Manuel, V.Chandrasekar and G.Britto Antony Xavier, Theory of Generalized $\alpha$-Difference Operator and its Applications in Number Theory, Advances in Differential Equations and Control Processes, 9(2) (2012), 141-155.

[13] S.K.Miller and B.Ross, An Introduction to the Fractional Calculus and Fractional Differential Equations, John Wiley and Sons, NewYork, 1993.

[14] K.S.Miller and B.Ross, Fractional Difference Calculus in Univalent Functions, Fractional Calculus and Their Applications(Koriyama, 1988),pp . 139-152, Horwood, Chichester, UK, 1989.

[15] N. R. O. Bastos, R. A. C. Ferreira, and D. F. M. Torres, Discrete-Time Fractional Variational Problems, Signal Processing, vol.91,no. 3,pp. 513524, 2011.

[16] R. A. C. Ferreira and D. F. M. Torres, Fractional h-difference equations arising from the calculus of variations, Applicable Analysis and Discrete Mathematics, 5 (2011), no.1,110-121.

[17] G. S. F. Frederico and D. F. M. Torres, A Formulation of Noether's theorem for fractional problems of the calculus of variations, Journal of Mathematical Analysis and Applications, 334 (2007), no.2,834-846.

[18] S.G.Samko, A.A.Kilbas, O.I.Marichev, Fractional integrals and derivatives, Translated from the 1987 Russian original,Gorden and Breach, Yverdon, 1993.

[19] Ronald E.Mickens, Difference Equations, Van Nostrand Reinhold Company, New York, 1990.

[20] J.Sabatier, O.P.Agrawal, J.A.Tenreiro Machado, Advances in fractional calculus, Springer, Dordrecht, 2007.

[21] Saber N.Elaydi, An Introduction to Difference Equations,2/e,Springer Verlag, 1999.

[22] Walter G.Kelley and Allan C. Peterson, Difference Equations, An Introduction with Applications, Academic Press Inc, Boston, 1991. 